\title{
Infancia en riesgo e infancia maltratada
}

\section{Montse Cusó}

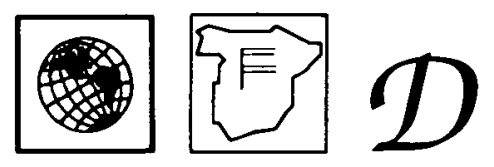

La autora parte de la necesidad de un elevado grado de competencia entre los profesionales de los servicios de bienestar relacionados con la infancia para detectar aquellas situaciones en las que confluyen distintos indicadores de riesgo, teniendo como punto de referencia los Derechos de la Infancia. Así mismo aborda el tema del maltrato infantil en sus distintas manisfestaciones, para proponer medidas que garanticen la atención a los niños y niñas que son víctimas de malos tratos.

\section{¿QUE ENTENDEMOS POR «INFANCIA EN RIESGO»?}

La expresión "Infancia en riesgo» se viene utilizando en la última década para referir aquella situación en que se encuentran los niños y niñas que viven inmersos en unas condiciones de vida que obstaculizan, perjudican o pueden perjudicar a corto y medio plazo su normal desarrollo como seres humanos especialmente vulnerables, entendido éste como un todo integral y armónico que envuelve y contiene elementos fisico-biológicos, psicológicos, emotivos, socio-afectivos, etc.

Cuando la evolución del niño en cualquiera de estos aspectos se ve amenazada o se halla ya comprometida por factores comprendidos en el entorno familiar y/o social inmediato, hablamos de «niño o niña en riesgo o en alto riesgo psico-social». A los mencionados factores se les puede medir mediante Indicadores de riesgo y solamente un riguroso análisis e interpretación de los mismos puede acercar a los profesionales que trabajan en la Protección a la Infancia a «medir», verificar, descartar o confirmar el riesgo real en que se halla un niño o una niña, y a menudo, un conjunto de niños y niñas.

Los Indicadores de riesgo, como fácilmente podemos deducir, nos indican en qué tipo y en qué grado se halla un niño o una niña en riesgo, pero no nos proporcionan una seguridad absoluta sobre la situación del niño o niña. Por este motivo, es de suma importancia la habilidad y competencia de los profesionales de los servicios de bienestar relacionados con la infancia en el análisis, la elaboración y la interpretación de los mismos. Podríamos decir, que deben ser capaces de "traducir», de «releer de otra forma» los indicadores presentes en cada caso, interrelacionándolos y contextualizándolos, siempre bajo el prisma de los Derechos de la Infancia.

Es importante que estos mismos profesionales, generalmente trabajando de forma sectorizada, identifiquen «entornos de riesgo» (Casas, 1994), además de "niños 
en riesgo», ya que pueden promover o colaborar en programas de ámbito comunitario que tengan el objectivo de disminuir el riesgo.

Los Indicadores de riesgo pueden ser clasificados según diversas categorías, de las cuales citaremos sólo dos, por considerarlas más clarificadoras.

De una parte, podemos clasificar los Indicadores de riesgo según si pertenecen a: a) el entorno familiar y/o social del niño o de la niña; b) si se hallan en el propio niño o niña; oc) si pertenecen al entorno físico-ambiental del mismo o la misma.

Citar o describir aquí los indicadores pertenecientes a cada una de las categorías superaría las posibilidades del presente artículo, pero sí creemos que es importante, aunque puedan ser de muchos conocidos, citar los más relevantes, ya sea por la gravedad que entrañan, o por la frecuencia con que se dan:

- niños y niñas que han sido víctimas de malos tratos físicos, psíquicos o sexuales en alguna ocasión.

- niños y niñas que han sido víctimas de situacions de abandono o negligencia con anterioridad.

- niños y niñas que tienen o han tenido algún hermano o hermana que ha sido víctima de algún tipo de maltrato, y/o que han tenido que ser tutelados por la institución competente.

- hijos e hijas de familias monoparentales, cuando a este hecho se suman otros factores de vulnerabilidad familiar.

- hijos e hijas de familias en las que ambos progenitores padecen algún tipo de disminución física o psíquica.

- niños y niñas que conviven con progenitores u otros adultos que sufren adicción a las drogas.

- niños y niñas con uno o ambos progenitores encarcelados.

- niños de otras etnias o razas, cuando a éste hecho se suman otros factores, como por ejemplo: exclusión social, insuficiencia de recursos económicos, marginación, estigmatización, aislamiento social, etc.

- niños y niñas que viven en un entorno en el que predominan la delincuencia, la falta de condiciones físicas para vivir dignamente, la falta de condiciones sociales para desarrollar su potencial relacional, la falta de redes sociales establecidas o informales, la marginalidad, etc.

De otra parte, podemos categorizar los Indicadores de riesgo según sean determinantes o circunstanciales. Llamamos factores de riesgo determinantes aquellos que por sí mismos, ya determinan una situación de riesgo, como por ejemplo, cualquier tipo de maltrato. Mientras que los indicadores circunstanciales serían aquellos que solamente indican una posible situación de riesgo si se dan en determinadas circunstancias o acompañados de otros indicadores que aumentan la vulnerabilidad y la fragilidad de la situación, como por ejemplo, el embarazo de una adolescente: sería un indicador de riesgo si a él se sumaran otros, como, por ejemplo, la falta de apoyo o el rechazo familiar, la disminución psíquica, etc.

\section{LOS MALOS TRATOS INFANTILES}

Podríamos llamar «maltrato infantil» a cualquier acción u omisión que vulnere alguno de los Derechos de la Infancia, y no faltaríamos a la verdad. De todas formas, que el término maltrato o malos tratos infantiles se aplica comúnmente a las acciones realizadas por los adultos que atentan contra la supervivencia o la integridad física de los menores.

Anteriores a este término, podemos encontrar: «el síndrome del niño apaleado» (Tardieu), «los niños mártires» (Bamatter), «el síndrome del abuso infantil» (Silver) etc. (Inglés, et al., 1991). 
En la Declaración de los Derechos de la Infancia adoptada por las Naciones Unidas el año 1959, reconocida anteriormente en la Declaración de los Derechos Humanos el año 1948, dice:

«El niño, por razón de su inmadurez mental y física, necesita atención y salvaguardas especiales, así como protección legal adecuada, tanto antes de su nacimiento, como después del mismo».

Y en la Convención sobre los Derechos del Niño de la Asamblea General de las Naciones Unidas el año 1989, se acuerda el compromiso de cumplir 54 Artículos con el objetivo de defender y proteger los derechos de los niños.

A pesar de todo ello, nos encontramos todavía bastante lejos de conseguir el cumplimiento de estos derechos, a pesar de haber sido firmados y ratificados por más de 150 países.

La Atención a la Infancia en un sentido amplio se da desde distintos ámbitos (salud, educación, etc.), pero a menudo cuando utilizamos esta expresión, nos referimos a la atención prestada a los niños y niñas que se hallan en situaciones más desventajosas o especialmente difíciles, que requiren una intervención directa del Estado. Los derechos son los mismos para todos los niños y niñas, pero sus necesidades varían según la vulneración de los mismos que afecte a su persona, ya sea por factores familiares, sociales, u otros.

Es decir, aquella atención pensada para los niños y niñas cuyos derechos están más vulnerados. A pesar de que esta priorización es fácilmente comprensible e incluso justificable, puede también constituir una trampa que nos lleve a considerar este estado de cosas como el deseable. Por ello, cada vez hay más voces que señalan que el camino a seguir debería pasar necesariamente por una atención normalizada y normalizadora ofrecida al conjunto social de la infancia, y no solamente a la parte más desfavorecida de la misma. En este sentido, es esperanzador y estimulante observar las nuevas Leyes Generales de Infancia aprobadas en algunas CC.AA. del estado español. (En Catalunya, está pendiente de aprobación la «Llei General de la Infancia»)

Existen numerosas definiciones del maltrato infantil, de entre las que destacaremos la que hizo el Comité de Ministros del Consejo de Europa, y posteriormente incluída en la «Recomanació (79) 17 de 13 de setembre de 1979 del Parlament de Catalunya»:

\footnotetext{
«Se consideran niños maltratados aquellos que han sido objeto de sevicias corporales, o que son víctimas de una falta de afecto, o de una crueldad mental, de tales caracterísitcas que comprometen su desarrollo físico, intelectual o afectivo, cuando estos actos son realizados por las personas que tienen a su cargo la guarda del niño o bajo cuya autoridad estan situados de forma temporal o permanente».
}

Según el estudio llevado a acabo por el Departament de Benestar Social de la Generalitat de Catalunya, publicado en el libro Els Maltractaments Infantils a Catalunya, en el que se realizó por vez primera en Catalunya un estudio estadístico, durante el año 1988 hubo 5.989 niños y niñas maltratados en Catalunya: el 68,8\% de los niños y niñas registrados, fueron maltratados por el padre, el $82 \%$ por la madre y el $12,3 \%$ por otros adultos. Cabe especificar que la proporción más elevada de casos de maltrato infantil perpretados por la madre tiene una primera explicación en el hecho de que éstas pasan un número muy superior de horas con sus hijos e hijas, y que son normalmente las «encargadas» de su educación.

\section{Tipología de los malos tratos a menores}

Hay diversas formas de clasificar los malos tratos infantiles, y de ellas exponemos la más clara y sencilla:

1. Maltrato Físico 


\section{0}

2. Maltrato psíquico o emocional

3. Maltrato sexual (Abuso sexual, Explotación sexual)

4. Negligencia o abandono

\section{Maltrato físico}

Recogemos aquí la definición de la National Society for the Prevention of Cruelty to Children (NSPCC), por considerarla muy precisa y clara: «Cuando los padres producen un daño físico, hieren o matan a un niño. Puede incluir los golpes, los zarandeos, los pellizcos, las quemaduras y los mordiscos. Incluye también dar sustancias venenosas, drogas inadecuadas o alcohol al niño, así como intentar ahogarlo por falta de aire o mediante el sumergimiento en agua» (Houghton, 1993). Sin embargo, Kempe matiza que las lesiones producidas deben requerir atención médica (tanto si ésta se le proporciona a la víctima, como si no) para ser consideradas como un maltrato.

\section{Algunas características de los perpretadores del maltrato físico}

La NSPCC especifica algunas de las características más comunes de los perpretadores del maltrato físico, de las que hemos seleccionado las siguientes:

- Padres más jóvenes que la media.

- Falta de redes de apoyo afectivas y sociales.

- Baja tolerancia al stress.

- Actitudes rígidas frente a la disciplina.

- Poco conocimiento de las necesidades evolutivas del niño, y unido a ello, espectativas inadecuadas para la edad de los mismos.

- Haber sido víctimas de maltrato en su infancia.

Una o más de estas características han sido encontradas en las situaciones de maltrato físico a un niño o niña, sin que ello implique, naturalmente, que quien muestra alguna de ellas pueda ser considerado un perpretados de maltrato potencial.

El maltrato físico, al ser frecuentemente apreciable a simple vista, puede inducir a error tanto a los profesionales que pueden detectarlo como a los que han de evaluarlo. De una parte, no es difícil caer en un análisis precipitado y a menudo simplista de la posible situación de maltrato, llevados por la impresión que nos causa cualquier daño físico a un menor. De otro, los profesionales no deben «quedarse» con la explicación o justificación de las heridas o hematomas que puedan dar los padres o cuidadores en un primer momento, aunque ésta pueda tranquilizarles, y tampoco deben posponer el estudio ulterior. Exponemos a continuación, unas sugerencias que creemos deben ser tenidas en cuenta por cuantos se hallan relacionados con niños y niñas, en el ámbito de la educación, la salud, el ocio, etc.:

1. Un análisis frable de la situación de maltrato físico debe ser capaz de compaginar la rapidez y celeridad, con la reflexión serena en equipo y una decisión elaborada y sopesada. Una primera intervención exploratoria debería realizarse en el tiempo más breve posible, ya que la vida del niño o niña puede estar en peligro.

2. La certeza de un maltrato físico solamente podemos tenerla después de la exploración y el diagnóstico médico.

3. Es de suma importancia escuchar las explicaciones de los padres y guardadores, y sobretodo las del niño o niña víctima del maltrato.

4. Al explorar una posible situación de maltrato físico de un niño o una niña, debemos también explorar a sus hermanos y hermanas, ya que muy a menudo pueden haber sido también víctimas del maltrato. (En el mencionado estudio, un $68,8 \%$ de los niños registrados tenían también hermanos maltratados, y un 23,4\% no tenían hermanos maltratados). 
5. Los profesionales no deben caer en la trampa de hacer suposiciones, ya que una herida que puede tener la apariencia de ser fruto de un maltrato, puede tener una explicación inocente, y viceversa.

6. El estudio de una posible situación de maltrato no debe nunca dejarse para «más adelante», ni siquiera cuando los indicios de los que disponemos son pocos y leves, y apuntan a un diagnóstico negativo. La vida de un niño, de una niña o de un hermano de los mismos está en peligro.

\section{Maltrato psíquico o emocional}

Escogemos de nuevo la definición de la NSPCC: «El hostigamiento verbal habitual del niño o niña mediante el desprecio, la crítica, la amenaza, y la falta de amor; de forma verbal y no verbal, del rechazo y de la inhibición.» (Houghton, 1993)

Este tipo de maltrato coincide a menudo con el maltrato físico, pero también se da en situaciones de niños y niñas que se hallan perfectamente atendidos desde el punto de vista de sus necesidades físicas.

El maltrato psíquico o emocional es mucho más sutil que el físico, y los expertos creen que es mucho más frecuente que aquel. Su falta de aparatosidad y el hecho de hallarse a menudo muy ligado a una determinada visión de los padres o cuidadores de lo que debe ser la educación de sus hijos e hijas, han determinado los tardíos y escasos estudios sobre el mismo, y la ignorancia de la población al respecto.

Existe una dificultad real en percibir y sentir que un niño está maltratado emocionalmente. Helfer \& Kempe, en su libro «The battered child» (1968), dicen: «Llegará un día quizás en que un médico podrá reconocer a un niño que sufre un maltrato emocional, pero este día todavía no ha llegado». Añaden más tarde, que el estudio del maltrato emocional se halla en una etapa inicial. Una de las razones por las que el maltrato emocional ha sido dejado de lado durante mucho tiempo, puede ser el hecho de que raramente requiere una intervención en crisis, de urgencia.

El maltrato psíquico es difícil de demostrar, a pesar de sus nefastos efectos en un ser en vías de desarrollo. A menudo son detectados mucho después de ser iniciados, cuando los síntomas de desequilibrio emocional se han hecho evidentes.

El maltrato emocional puede tener muchas formas: violencia verbal y física entre los padres, niños no deseados que son percibidos por sus padres como una carga difícilmente tolerable, padres que imponen normas de conducta muy estrictas, hijos e hijas de padres separados o divorciados que están continua y repetidamente sometidos a un conflicto de lealtades o a los que se les priva del contacto con uno de los progenitores, niños y niñas que viven bajo la presión sin tregua de conseguir buenos resultados escolares de forma obsesiva, niños y niñas cuya intimidad es vulnerada por sus padres o cuidadores, y muchos más.

Algunos indicadores de maltrato psíquico

- pérdida de peso sin razones médicas.

- aislamiento social.

- inhibición.

- confusión del niño sobre su vida y la de sus padres.

- inestabilidad emocional originada por diversas causas: cambios repetidos de adultos con los que convive (padres y madres inestables con parejas sucesivas), etc.

- trastornos del lenguaje y dificultades de aprendizaje.

- reacciones histéricas.

- conductas extremas: muy pasivo, y acomodaticio, agresivo y exigente.

Este tipo de maltrato no provoca escándalos ni investigaciones periodísticas, pero inflige un gran sufrimiento y graves crisis en el niño y en el adolescente.

Las causas del maltrato psíquico son generalmente factores de vulnerabilidad de los padres: padres muy jóvenes, padres solos, padres adolescentes muy centrados en 
sí mismos y en sus propias necesidades, padres inmaduros con dificultades de ajuste a la vida adulta, dificultades económicas añadidas a otros factores de riesgo, ausencia de orientación y apoyo social a los padres, etc.

\section{Maltrato sexual ( Abuso sexual, Explotación sexual)}

Hemos utilizado dos definiciones que nos han parecido en cierto modo complementarias y de éste modo clarificadoras respecto de un tema tan complejo como el maltrato o abuso sexual.

Kempe lo define de esta forma: « La implicación de niños, niñas y adolescentes dependientes, e inmaduros en cuanto a su desarrollo, en actividades sexuales que no comprenden plenamente y para las cuales son incapaces de dar un consentimiento informado.»

Y la NSPCC lo hace como sigue: «Cuando los niños o niñas son víctimas de abuso sexual por parte de los adultos, que los utilizan para satisfacer sus propias necesidades sexuales. Puede incluir el acto sexual, y también las caricias, la masturbación, el sexo oral y el hecho de mostrar o exponer a los niños y niñas material pornográfico, incluídos los vídeos.» (Houghton, 1993)

Se utiliza también el término «explotación sexual», al considerar que el abuso del que son víctimas estos menores les priva del control sobre sus propios cuerpos (Kempe \& Kempe).

El maltrato sexual es mucho más difícil de detectar que otros tipos de maltrato, debido a que no deja señales físicas aparentes (con excepción del perpretado con violencia). Si a ésto se añade el hecho de que en la mayoría de casos, los menores se hallan amenazados por el autor del maltrato, si rompen el «secreto» entre los dos; y los tabúes relacionados con el tema sexual en general, podemos intuir que la mayoría de situaciones de maltrato sexual infantil no salen a la luz.

Por este motivo, el estudio de una posible situación de maltrato sexual, entraña una gran complejidad, que requerirá una preparación especializada de los profesionales encargados de evaluar las situaciones de vulneración de los derechos de los niños y niñas, y una atenta supervisión de su trabajo. A menudo, no será necesario tomar medidas inmediatas de protección (con excepción de los casos en que el presunto perpretador y la víctima estén conviviendo en el momento en que se realiza el estudio), pero éste deberá iniciarse sin dilaciones, ya que el niño o la niña pueden necesitar ayuda urgente especializada. Las víctimas de este tipo de maltrato se sienten indefensas, culpables y amenazadas, sobre todo en el caso de las víctimas de incesto, que añaden a su situación la rabia contra su padre, y más virulenta todavía, contra su madre por no haberlas protegido.

Ya en el inicio del estudio, el profesional deberá hacer frente a las primeras dificultades para esclarecer la situación:

- En primer lugar, los presuntos perpretadores del maltrato, los padres y madres, los padrastros y las madrastras, los abogados defensores de los primeros, los medios de comunicación, etc., pretenderán convencerlos de la imposibilidad de que hayan sucedido los hechos relatados o denunciados por las víctimas, y la tendencia de los niños y niñas a inventarse «estas cosas» (Houghton, 1993).

- Más adelante, y sobre todo en el caso de que el maltrato se dé en el núcleo familiar, se encontrará con la confabulación de la familia para encubrir los hechos. A menudo los distintos miembros de la familia «saben» de una u otra forma lo que está sucediendo, pero también saben que si se descubre el serreto tan bien guardado, se va a ir al garete el «status quo» familiar. (Recordamos, por ejemplo, a la madre de una chica que había sido víctima de incesto continuado durante años, expresar su indignación, porque la revelación de su hija, le había dejado sin hombre y sin el salario del mismo).

- De la misma forma, deberá afrontar los sentimientos de repugnancia o de rechazo que le pueda producir abordar este problema, la tentación de subestimarlo e incluso la de negarlo. Es especialmente sorprendente observar como, en ocasiones, en 
una situación de posible maltrato sexual, al analizar los indicadores de riesgo hallados al respecto, la conclusión a la que llega el equipo de profesionales encargado del estudio es de que no ha existido tal abuso, mientras que analizados los mismos con la distancia por un supervisor y el mismo equipo, la conclusión a la que se llega es claramente la opuesta. Es decir, los indicadores están indicando que Sí existe una situación de maltrato sexual, pero el profesional o el equipo de profesionales no ba sabido o no ha podido leerlo!. Este hecho se produce a causa de una negación a aceptar una posible interpretación positiva, por causas diversas: el poco conocimiento del tema, la escasa concienciación sobre el mismo, la repugnancia y el desagrado que producen, los valores internalizados por el profesional en edades tempranas, las difíciles decisiones que deberá tomar si diagnostica el maltrato, el temor a equivocarse a pesar de haber reflexionado cuidadosamente, la crisis en la que entrará la familia y las posibles consecuencias de la misma en los diferentes miembros, la seducción del presunto perpretador del maltrato, etc.

Respecto a los indicadores que pueden sugerir que nos encontramos ante una situación de maltrato sexual, C.J. Hobbs y J. M. Wynne, del Hospital Universitario de St. James y Enfermería General, de Leeds (Gran Bretaña) describieron unos, a mi parecer, exhaustivos y precisos indicadores, que superan las posibilidades de inclusión en el presente artículo, pero que pueden ser de gran ayuda para aquellos profesionales que deseen especializarse en el tema.

Características de los perpretadores del maltrato sexual

Una vez más nos remitiremos a la experiencia de la NSPCC respecto al maltrato, y resumiremos algunas de las características que se han observado en los perpretadores del abuso sexual:

- Tienden a ser seductores.

- Calculan el abuso que van a cometer con premeditación.

- Tienen como objetivo la penetración, aunque no siempre llegan a ella.

- Escogen niños o niñas vulnerables. Es decir, no escogen a la más bonita, sino a la más indefensa.

- Generalmente crean vínculos afectivos con la víctima.

Los expertos creen que el maltrato sexual es adictivo, y como tal, raramente se puede llegar a la total curación. De todas formas, algunos tratamientos tienen resultados muy positivos, en cuanto posibilitan un grado muy alto de auto-control por parte del perpretador-paciente.

Respecto a cómo prevenir el maltrato sexual, sobre todo el que se produce en medios extrafamiliares, en algunos países europeos se han confeccionado programas escolares para defenderse de los ataques sexuales, que lejos de alarmar a los menores, les preparan para evitarlos, para afrontarlos si se dan, y para denunciarlos si no han podido ser evitados.

\section{Negligencia 0 abandono}

Llamamos negligencia o abandono a aquella situación que padecen un importante número de niños y niñas que se hallan en el espacio situado entre una situación de no atención adecuada a la de maltrato por desatención. En este contexto denominamos «atención» a todo lo que hace referencia a la nutrición, la higiene, la salud, la protección contra los riesgos físicos, etc.

El Dr. David H. Skuse la define como sigue: «La falta de cuidados físicos, y el hecho de no satisfacer las necesidades de desarrollo del niño desde una perspectiva de estimulación cognitiva».

Algunas características de la negligencia en el cuidado de los niños y niñas (Houghton, 1993)

- Físicas: falta de desarrollo pondo-estatural, pequeñas infecciones recurrentes y persistentes, ausencia de control médico a través de los circuitos normalizados, visitas frecuentes a los hospitales por la vía de urgencia. 

desarrollo.

- De desarrollo: retraso generalizado sin llegar a los mínimos estándares de

- De conducta: ansiedad, falta de respuesta a los estímulos físicos o afectivos, inhibición, poca o nula vinculación con los padres o cuidadores, depresión, apatía.

Este tipo de maltrato implica generalmente una actuación inadecuada de los padres o cuidadores respecto del niño o niña, ya sea por incapacidad personal, por acumulación de problemas en el núcleo familiar (por ejemplo, en el caso de familias muy numerosas, unido a insuficentes recursos personales $y / 0$ materiales, imposibilidad de «llegar a todo»), o bien por un concepto erróneo de lo que debe ser una dieta correcta.

Los profesionales que han de evaluar estas situaciones, en ocasiones «fronterizas», se enfrentan a un difícil reto: cómo intervenir en ellas para evitar, de un lado, una separación de los niños de sus padres o cuidadores (no adecuada en general para estos casos, y a menudo innecesaria), y del otro, asegurarse de que la situación no solo no empeora, sino que no se cronifica. Es un reto difícil, pero demasiadas veces nos hemos encontrado ante adolescentes con una historia de abandono largamente sufrido, que les han dejado graves e irreparables secuelas: falta de confianza en sí mismos, mala relación social, poca capacidad para solucionar sus problemas, y otras, que pueden llevarlos a la delincuencia. Trabajar con una familia para mejorar, paliar o disminuir la desatención de los menores de la familia, es trabajar para la prevención de la delincuencia.

\section{LA ATENCION A LOS NIÑNOS Y NIÑAS VICTIMAS DE MALOS TRATOS}

Desafortunadamente se dan situaciones en las que los derechos de los niños y los derechos de sus propios padres en encuentran en conflicto. Ésto lo saben y lo sufren los profesionales que trabajan en el campo de la atención a la infancia, ya que los objectivos de proteger al niño por una parte y de preservar las unidades familiares por otra, implican una gran complejidad: ¿Cómo pueden los profesionales intervenir en situaciones familiares de forma que el niño o niña sea protegido, que se preserve la integridad de la familia, y que se reduzca al mínimo la "intrusión" del Estado? (Mayer, 1983). La creación de equipos interdisciplinares especializados en el estudio, evaluación y tratamiento de las situaciones de maltrato infantil (en Cataluña, los EALA's, creados en funcionamiento desde 1988) (Decreto 338/86 de la Gen. de Cat.) sale al paso de esta dificultad. El trabajo en equipo permite la multiplicación de las percepciones y por tanto, de los análisis, y evita en parte, la posible arbitrariedad y subjetividad de la valoración individual, facilitando su control.

Es importante encontrar formas de proteger a los menores sin aumentar los sentimientos de aislamiento social y de impotencia que padecen muchos padres infligidores de malos tratos.

La ignorancia y la falta de sensibilización respecto a los derechos de los niños generan duras críticas hacia los estamentos y los profesionales que trabajan para garantizar que estos derechos no sean gravemente vulnerados, y sobre todo un gran rechazo hacia las actuaciones concretas que se toman al respecto. De esta forma, consciente $o$ inconscientemente, se priorizan de forma habitual los derechos de los adultos a los de los niños. Solamente cuando un niño o una niña ha muerto, o ha sido brutalmente golpeado o quemado o es víctima de abuso sexual manfiestamente reconocido, la opinión pública y los medios de comunicación se hacen eco de la situación. En estos casos, las actitudes de los ciudadanos son durísimas al respecto, pidiendo incluso la pena de muerte o el encarcelamiento de por vida de los perpretadores del maltrato. Pero es antes de que ocurra un daño irreparable cuando los profesionales deben tomar difíciles decisiones, a veces preventivas. Generalmente, las actitudes de rechazo de la gente hacia la intervención del Estado y de los profesionales, encierra una infravalomción del sufrimiento del niño, restos todavía de la vieja idea de que los hijos son pro- 
piedad de los padres, y de que están bajo su única responsabilidad, y por lo tanto se justifica en la opinión de que cada progenitor debe educar a su hijo a su manera.

Por otra parte, intervenir sobre algo que «todavía no ha ocurrido», significa intervenir sobre algo invisible a los ojos de los que no son expertos en el tema, ya que las probabilidades o no de que sucedan, proviene de las evidencias acumuladas por los profesionales y de los análisis de los investigadores. Es decir, los profesionales solamente están socialmente legitimados para intervenir en situaciones de alto riesgo psico-social, cuando ya se ha producido un maltrato evidente (Casas, 1993).

Las actitudes de rechazo a la intromisión del Estado permiten que todavía nos encontremos con padres, madres, abuelos, maestros, médicos, etc. que conozcan situaciones de niños gravemente maltratados y no lo denuncien a los organismos competentes: en un 40,9\% de casos de maltrato infantil detectados en la escuela, ésta no derivó el niño o niña a un servicio especializado, sino que lo solucionó con sus propios medios; en el mismo estudio describen como los pediatras afirman no haber iniciado ninguna actuación en un $23 \%$ de los casos de maltrato que habían detectado (Inglés et al., 1991).

A pesar de que los estudios de los niños presuntamente víctimas de malos tratos requiere una atención especializada realizada por profesionales expertos en el tema, y sujetos a supervisión, la tarea de detectar y explorar los malos tratos en el momento de ser apreciados, es un trabajo en que deberían estar implicados todos los servicios de bienestar. El creciente interés y sensibilización de los colectivos que estan directa o indirectamente relacionados con los niños y niñas nos hace ser positivos al respecto, ya que el trabajo en red se está llevando ya a cabo, con el consiguiente enriquecimiento de todos los profesionales, y el beneficio de haber aumentado significativamente la detección de los mismos, y la eficacia y eficiencia en los diagnósticos. No podemos decir lo mismo en lo referente al tratamiento y seguimiento de las familias y los niños después del estudio y de la toma de medidas si es necesario, ya que hace falta más preparación específica por parte de los profesionales de base, así como ratios mucho más favorables en el caso de los equipos especializados, para poder intervenir en las familias con resultados positivos. Por otra parte, existen en nuestro país muy pocos equipos de tratamiento especializados para situaciones de maltratos específicos (por ejemplo, el incesto). Sin un tratamiento específico por un equipo de terapéutas y un seguimiento/acompañamiento cetcano por el EAIA o equipo correspondiente, se hace muy difícil que el niño o la niña pueda retornar a su casa, sin poner en peligro su integridad.

La creación de servicios educativos y lúdicos de ayuda y apoyo a padres y madres, ya sea en forma de «Casas de familias», de grupos de padres, de escuelas de padres, etc., así como propiciar unos sistemas de acceso a la vivienda y al trabajo más asequibles, evitarían un buen número de malos tratos, ya que, a pesar de que nadie sabe exactamente como se transmite la aptitud para ser padre, la ignorancia de las necesidades de los niños, la falta cada vez más alarmante de redes de apoyo afectivo y social primarias y informales, el enervante efecto de una situación de pobreza continuada, contribuye a aumentar el número de ellos.

Para terminar, hacer mención de un hecho que no podemos soslayar: los malos tratos infantiles se dan en todos los estratos de la sociedad, pero la atención (y por ende, el control a las familias) a los niños y niñas víctimas de malos tratos o en riesgo de serlo se refiere, llega solamente a las capas más desfavorecidas de la sociedad, ya que solamente hacen uso de los circuitos de los servicios sociales, los individuos y familias que en mayor o menor medida padecen una problemática socio-económica grave. Los circuitos que siguen los casos de niños y niñas víctimas de malos tratos de estratos sociales más elevados, pasan generalmente por los servicios privados, que no están sujetos a control, y que carecen a menudo de una adecuada sensibilización respecto al tema de los malos tratos. De esta forma, el Estado «interfiere» en los núcleos familiares más vulnerables, añadiendo una sensación de injusticia a los padres/madres sujetos a su intervención, que en nada beneficia, el trabajo de rehabilitación y superación de sus handicaps, que deben realizar para no perder a sus hijos. La otra cara de la moneda 
la representan todos aquellos niños y niñas que por pertenecer a clases sociales medias o elevadas, y ser víctimas de tipos de maltrato más sutiles, no tienen oportunidad de ser atendidos que podrían ayudarles a ellos y a sus padres o cuidadores.

\section{Referencias}

CASAS I AZNAR, F. (1994). «Prevenció i Qualitar de vida dels Infants». Forum. Generalitat de Catalumya, Departament de Benestar Social. Barcelona, 1, 41- 42.

Cusó i TOReLLó, M. «L'estudi de les situacions d'infants en risc». Ponencia presentada en el Congrés Europeu d'Atenció a la Infància. Barcelona, 1992.

GOLDSTEIN, FREUD y SOLNIT (1982). Beyond the best interest of the child.. Londres: Burnett Books LTD.

HOBBS, C.J. y WYNNE, J.M. (1987). «Management of sexual abuse». Archives of Disease in Childhood. London: British Medical Association.

Houghton, J.W. "Social Intervention in Child Protection I i II». Cursos impartits al 1993 - 1994. Barcelona: ICESB.

INGLÉs I PrATS, A., et al. (1993). Els maltractaments infantils a Catalunya. Generalitat de Catalunya. Departament de Benestar Social.

KeMPE, R.S. y KeMPE, C.H. (1979). Niños maltratados. Madrid: Morata.

MAYER, B. (1984). Conflict resolution in cbild protection and adoption. Document policopiat

\section{Infancia en riesgo e infancia maltratada \\ Montse Cusó \\ CL\&E, 1995, 27, pp. 87-96}

Resumen: Un número importante de niños y niñas se encuentra en situación de riesgo. Podríamos decirlo de otro modo, como que los derechos de estos niños y niñas se hallan en riesgo de ser vulnerados. De otra parte, otros niños son víctimas de una vulneración clara de sus derechos: son los niños y niñas víctimas de algún tipo de maltrato: físico, psíquico, sexual o negligencia (abandono). La detección de los mismos ha dado un paso importante en los últimos años en nuestro país, pero no así el tratamiento especializado de los padres/madres infligidores de maltrato. En el terreno de la prevención, se hace necesario ofrecer a los padres y madres más vulnerables, espacios de apoyo psico-afectivo, y educativo que evitaría un buen numero de situaciones de maltrato.

Datos sobre la autora: Montse Cusó Torelló es Diplomada en Trabajo Social. Coordinadora de la Unidad de Acogimientos Familiares Simples de la Direcció General d'Atenció a la Infancia del Departament de Benestar Social de la Generalitar de Catalunya. Anteriormente había trabajado en el Equipo Técnico Multidisciplinar, en la Direcció General de Protecció i Tutela de Menors, del Departament de Justícia. Es miembro del Equipo de Redacción de la Revista de Treball Social (RTS).

Dirección: Dirección General de Atención a la Infancia, Departament d'Acolliment i Adopcions. Edifici Palau del Mar. Plça Pau Vila 1. 08003 Barcelona.

(c) PERMISOS PARA CITAR O REPRODUCIR EN OTRAS FUENTES: Se pueden citar libremente hasta 500 palabras. Para reproducir una porción de texto mayor, figuras o ilustraciones, se deberá pedir permiso por escrito a la revista, especificando el uso al que se destina el texto. En todos los casos, se deberá citar el copyright de $C L \& E$. En el caso de artículos o textos que hayan sido a su vez reproducidos en $C L \& E$ los interesados deberán dirigirse tanto a los detentadores del copyright original como a $C L \& E$, en el caso de que se quiera hacer uso de la traducción. FOTOCOPIAS: Para todo lo relacionado con el uso mediante fotocopia del material de esta revista, deberán dirigirse a: CEDRO, C/ José Marañón, 10, 3. Izda. Tel. 5941575 . Fax 4453567 